\title{
Preeclampsia and diuresis criteria: our experience at the University of lowa Hospitals and Clinics
}

\author{
Kim Naruko-Stewart, $\mathrm{MD}^{1}$ \\ Keywords: diuresis, magnesium sulfate, postpartum period, pre-eclampsia, pregnancy
}

\section{Objective}

This study was primarily designed to evaluate two hypotheses:

1. For those women with mild preeclampsia, ceasing magnesium sulfate therapy after diuresis (see above for criteria) does not increase the risk of having to re-start magnesium sulfate therapy.

2. For those women with severe preeclampsia, using the above diuresis criteria to allow cessation of magnesium sulfate prior to 24 hours postdelivery would not put them at any increased risk of having to re-start magnesium sulfate therapy.

${ }^{1}$ Department of Obstetrics and Gynecology, Carver College of Medicine, University of lowa Hospitals and Clinics, lowa City, IA, 52242

\section{Background}

Preeclampsia is a multisystem disorder that complicates approximately $5-8 \%$ of pregnancies. Although outcomes are generally good, preeclampsia is a major cause of morbidity and mortality for a woman and her unborn child. Preeclampsia and eclampsia probably account for more than 50,000 maternal deaths a year.

The use of magnesium sulfate for seizure prophylaxis in women with preeclampsia was well established by the Magpie Trial in $2002 .{ }^{1}$

It is known that the clinical manifestations of preeclampsia are a result of widespread vasoconstriction. It is therefore thought that the onset of diuresis may signal a reversal of this vasoconstriction, and thus resolution of the disease. Here at the University of lowa Hospitals and Clinics (UIHC), women with mild preeclampsia are treated with post-

Please cite this paper as: Naruko-Stewart K. Preeclampsia and diuresis criteria: our experience at the University of lowa Hospitals and Clinics. Proc Obstet Gynecol. 2011;2(2):Article 14 [3 p.]. Available from: http://ir.uiowa.edu/pog/. Free full text article.

Corresponding author: Kim Naruko-Stewart, Department of Obstetrics and Gynecology, University of lowa, 51244 PFP, 200 Hawkins Drive, lowa City, IA 42242. 
partum magnesium sulfate until specific diuresis criteria are met (200 $\mathrm{ml}$ urine/hour for four consecutive hours) or for a maximum of 24 hours post-delivery. Women with severe preeclampsia are routinely given 24 hours of magnesium sulfate therapy post-delivery regardless of urine output.

\section{Methods}

This retrospective cohort study was conducted at the University of lowa Hospitals and Clinics (UIHC) to validate our diuresis criteria and determine if there was a trend for diuresis that was dependent on the type of preeclampsia. UIHC institutional review board approval was obtained prior to the study. Medical records were selected for review based on billing codes for hypertension and preeclampsia; we report data from patients discharged from UIHC between March, 2004 and May, 2008. Medical records of this cohort of patients were utilized to extract pertinent data including demographics, medical and obstetrical history data, labor characteristics, urine outputs during magnesium sulfate therapy and newborn characteristics.

The use of magnesium sulfate at the University of lowa Hospital and Clinics is as follows: Women diagnosed with mild preeclampsia receive 2 grams of magnesium sulfate per hour until diuresis criteria are met (see above for details) or they reach 24 hours post-delivery at which point it is discontinued. Women diagnosed with severe preeclampsia receive 2 grams of

Preeclampsia and diuresis criteria magnesium sulfate per hour for 24 hours post-delivery regardless of urine output. Any decision to restart magnesium sulfate was made by the resident and attending physician that were on Labor and Delivery at that time and included patient symptoms consistent with preeclampsia as well as uncontrolled high blood pressures. Due to the fact that eclampsia is a rare event, we used the re-initiation of magnesium sulfate as our primary outcome.

\section{Results}

There were no surprises in terms of differences that were significantly different including: gestational age at delivery, urine dip on admission, aspartate aminotransferase (AST) and alanine aminotransferase (ALT) on admission, 24 hour urine protein, birth weight and Apgar scores at 1 and 5 minutes. The time to diuresis was significantly different.

Rates of re-administration of magnesium sulfate were compared between the patients with mild preeclampsia, the patients with severe preeclampsia that did not diuresis and the patients with severe preeclampsia that would have met our diuresis criteria. There is a trend toward a significant difference between those patients with mild preeclampsia and those with severe preeclampsia who never met diuresis criteria. There was no difference in the rate of re-initiating magnesium sulfate when comparing those patients with mild preeclampsia and those with severe preeclampsia who would have met diuresis criteria. 


\section{Conclusion}

This study suggests that the diuresis curves for those women with mild preeclampsia and severe preeclampsia do not vary significantly after the first 5 hours. It also appears that the rate of reinitiation of magnesium sulfate is low for those women diagnosed with mild preeclampsia and treated using the UIHC protocol for magnesium sulfate administration post-delivery.

We were able to separate the women with severe preeclampsia into two groups, one of which would have met our diuresis criteria prior to 24 hours and the other of which would not have met diuresis criteria at 24 hours post-delivery. It is interesting to note that when these groups were compared, there is a trend toward a significant difference between those patients with mild preeclampsia and those with severe preeclampsia who never met diuresis criteria. It is also interesting to note that there was no significant difference in the rate of restarting magnesium sulfate for women with mild preeclampsia and those with severe preeclampsia who would have met diuresis criteria. It seems reasonable to conclude that it may not be necessary to require 24 hours of magnesium sulfate for those women with severe preeclampsia who meet diuresis criteria.

\section{References}

${ }^{1}$ Altman D, Carroli G, Duley L, Farrell B, Moodley J, Neilson J, Smith D; Magpie Trial Collaboration Group. Do women with preeclampsia, and their babies, benefit from magnesium sulphate? The Magpie Trial: a randomised placebo-controlled trial. Lancet. 2002 Jun 1;359(9321):1877-90. 